\title{
Binuclear Cyclopentadienylmetal Nitrosyls of Iron, Cobalt, and Nickel: Comparison with Related Carbonyl Derivatives
}

\author{
Hongyan Wang, ${ }^{a, b}$ Yaoming Xie, ${ }^{b}$ R. Bruce King, ${ }^{b}$ and Henry F. Schaefer III ${ }^{b}$ \\ Atomic and Molecular Physics Institute, Sichuan University \\ Sichuan 610065, P. R. China \\ and \\ Department of Chemistry and Center for Computational Chemistry \\ University of Georgia, Athens, Georgia 30602, USA
}

\section{Supporting Information}

Table S1. Harmonic vibrational frequencies (in $\mathrm{cm}^{-1}$ ) and infrared intensities (in parentheses, in $\mathrm{km} / \mathrm{mol})$ for the structure $\mathbf{I a}$ of $\mathrm{Cp}_{2} \mathrm{Fe}_{2}(\mu-\mathrm{NO})_{2}$ with $\mathrm{C}_{2 \mathrm{v}}$ symmetry

Table S2. Harmonic vibrational frequencies (in $\mathrm{cm}^{-1}$ ) and infrared intensities (in parentheses, in $\mathrm{km} / \mathrm{mol}$ ) for the structure $\mathbf{I b}$ of $\mathrm{Cp}_{2} \mathrm{Fe}_{2}(\mathrm{NO})_{2}$ with $\mathrm{C}_{2 \mathrm{~h}}$ symmetry

Table S3. Harmonic vibrational frequencies (in $\mathrm{cm}^{-1}$ ) and infrared intensities (in parentheses, in $\mathrm{km} / \mathrm{mol})$ for the structure IIa of $\mathrm{Cp}_{2} \mathrm{Fe}_{2}(\mu-\mathrm{NO})$ with $\mathrm{C}_{2 \mathrm{v}}$ symmetry

Table S4. Harmonic vibrational frequencies (in $\mathrm{cm}^{-1}$ ) and infrared intensities (in parentheses, in $\mathrm{km} / \mathrm{mol}$ ) for the structure IIb of $\mathrm{Cp}_{2} \mathrm{Fe}_{2}(\mathrm{NO})$ with $\mathrm{C}_{\mathrm{s}}$ symmetry

Table S5. Harmonic vibrational frequencies (in $\mathrm{cm}^{-1}$ ) and infrared intensities (in parentheses, in $\mathrm{km} / \mathrm{mol})$ for the structure III of $\mathrm{Cp}_{2} \mathrm{Co}_{2}(\mu-\mathrm{NO})_{2}$ with $\mathrm{C}_{2 \mathrm{v}}$ symmetry

Table S6. Harmonic vibrational frequencies (in $\mathrm{cm}^{-1}$ ) and infrared intensities (in parentheses, in $\mathrm{km} / \mathrm{mol})$ for the structure IVa of $\mathrm{Cp}_{2} \mathrm{Co}_{2}(\mu-\mathrm{NO})$ with $\mathrm{C}_{2 \mathrm{v}}$ symmetry

Table S7. Harmonic vibrational frequencies (in $\mathrm{cm}^{-1}$ ) and infrared intensities (in parentheses, in $\mathrm{km} / \mathrm{mol})$ for the structure $\mathbf{I V b}$ of $\mathrm{CpCo}_{2}(\mu-\mathrm{Cp})(\mathrm{NO})$ with $\mathrm{C}_{\mathrm{s}}$ symmetry Table S8. Harmonic vibrational frequencies (in $\mathrm{cm}^{-1}$ ) and infrared intensities (in parentheses, in $\mathrm{km} / \mathrm{mol}$ ) for the structure $\mathbf{V}$ of $\mathrm{Cp}_{2} \mathrm{Ni}_{2}(\mu-\mathrm{NO})_{2}$ with $\mathrm{C}_{1}$ symmetry

Table S9. Harmonic vibrational frequencies (in $\mathrm{cm}^{-1}$ ) and infrared intensities (in parentheses, in $\mathrm{km} / \mathrm{mol}$ ) for the structure $\mathrm{VI}$ of $\mathrm{Cp}_{2} \mathrm{Ni}_{2}(\mu-\mathrm{NO})$ with $\mathrm{C}_{2 \mathrm{v}}$ symmetry

Table S10. Harmonic vibrational frequencies (in $\mathrm{cm}^{-1}$ ) and infrared intensities (in parentheses, in $\mathrm{km} / \mathrm{mol}$ ) for the structure VIIa of $\mathrm{Cp}_{2} \mathrm{Ni}_{2}(\mu-\mathrm{CO})_{2}$ with $\mathrm{C}_{2 \mathrm{~h}}$ symmetry 
Table S11. Harmonic vibrational frequencies (in $\mathrm{cm}^{-1}$ ) and infrared intensities (in parentheses, in $\mathrm{km} / \mathrm{mol}$ ) for the structure VIIb of $\mathrm{Cp}_{2} \mathrm{Ni}_{2}(\mu-\mathrm{CO})_{2}$ with $\mathrm{C}_{2 \mathrm{~h}}$ symmetry

Table S12 Harmonic vibrational frequencies (in $\mathrm{cm}^{-1}$ ) and infrared intensities (in parentheses, in $\mathrm{km} / \mathrm{mol}$ ) for the structure VIIc of triplet $\perp-\mathrm{Cp}_{2} \mathrm{Ni}_{2}(\mathrm{CO})_{2}$ with $\mathrm{C}_{2 \mathrm{~h}}$ symmetry

Table S13. Harmonic vibrational frequencies (in $\mathrm{cm}^{-1}$ ) and infrared intensities (in parentheses, in $\mathrm{km} / \mathrm{mol}$ ) for the structure VIIIa of singlet $\mathrm{Cp}_{2} \mathrm{Ni}_{2}(\mu-\mathrm{CO})$ with $\mathrm{C}_{2 \mathrm{v}}$ symmetry

Table S14. Harmonic vibrational frequencies (in $\mathrm{cm}^{-1}$ ) and infrared intensities (in parentheses, in $\mathrm{km} / \mathrm{mol}$ ) for the structure VIIIb of singlet $\mathrm{Cp}_{2} \mathrm{Ni}_{2}(\mathrm{CO})$ with $\mathrm{C}_{\mathrm{s}}$ symmetry

Table S15. Harmonic vibrational frequencies (in $\mathrm{cm}^{-1}$ ) and infrared intensities (in parentheses, in $\mathrm{km} / \mathrm{mol}$ ) for the structure VIIIc of triplet $\mathrm{Cp}_{2} \mathrm{Ni}_{2}(\mathrm{CO})$ with $\mathrm{C}_{\mathrm{s}}$ symmetry

Table S16. Cartesian coordinates for the 15 structures discussed in this paper. 
Table S1. Harmonic vibrational frequencies $\left(\right.$ in $\mathrm{cm}^{-1}$ ) and infrared intensities (in parentheses, in $\mathrm{km} / \mathrm{mol}$ ) for the structure $\mathbf{I a}$ of $\mathrm{Cp}_{2} \mathrm{Fe}_{2}(\mu-\mathrm{CO})_{2}$ with $\mathrm{C}_{2 \mathrm{v}}$ symmetry

\begin{tabular}{|c|c|c|c|c|c|}
\hline & B3LYP & BP86 & & B3LYP & BP86 \\
\hline \multirow[t]{21}{*}{ a1 } & $53(1)$ & $55(2)$ & b1 & $17 \mathrm{i}(0)$ & $8 \mathrm{i}(0)$ \\
\hline & $133(2)$ & $126(1)$ & & $112(2)$ & $112(2)$ \\
\hline & $200(0)$ & $202(0)$ & & $344(25)$ & $342(24)$ \\
\hline & $363(8)$ & $366(8)$ & & $450(0)$ & $431(0)$ \\
\hline & $468(0)$ & $465(0)$ & & $590(0)$ & $558(0)$ \\
\hline & $551(0)$ & $542(0)$ & & $624(7)$ & $604(6)$ \\
\hline & $593(0)$ & $564(0)$ & & $840(7)$ & $802(6)$ \\
\hline & 647(29) & $601(22)$ & & $850(14)$ & $817(12)$ \\
\hline & $832(0)$ & 796(0) & & 912(1) & 864(3) \\
\hline & $841(1)$ & $802(0)$ & & $1022(14)$ & 988(15) \\
\hline & $850(0)$ & $819(0)$ & & 1073(0) & $1040(0)$ \\
\hline & $916(1)$ & $867(2)$ & & 1273(0) & $1222(0)$ \\
\hline & 1024(26) & 991(24) & & 1398(0) & $1356(0)$ \\
\hline & 1072(0) & $1040(0)$ & & $1455(5)$ & 1401(4) \\
\hline & 1138(0) & $1106(0)$ & & 1607(1078) & $1566(811)$ \\
\hline & 1399(0) & $1360(0)$ & & $3239(0)$ & $3161(0)$ \\
\hline & 1457(4) & 1403(4) & & $3254(0)$ & $3175(0)$ \\
\hline & 1661(0) & 1604(0) & b2 & $170(0)$ & $166(0)$ \\
\hline & 3241(0) & $3162(0)$ & & $248(8)$ & $246(7)$ \\
\hline & 3252(1) & $3174(2)$ & & $365(2)$ & $364(1)$ \\
\hline & 3264(0) & $3185(0)$ & & $404(0)$ & $405(0)$ \\
\hline \multirow[t]{16}{*}{$\mathrm{a} 2$} & $17 \mathrm{i}(0)$ & $3(0)$ & & $586(4)$ & $558(2)$ \\
\hline & $153(0)$ & $152(0)$ & & $716(240)$ & $732(188)$ \\
\hline & $300(0)$ & $298(0)$ & & $832(80)$ & 798(27) \\
\hline & $444(0)$ & $423(0)$ & & $841(7)$ & 802(16) \\
\hline & $591(0)$ & $561(0)$ & & $850(25)$ & $821(51)$ \\
\hline & $634(0)$ & $602(0)$ & & 914(4) & $865(9)$ \\
\hline & $841(0)$ & $804(0)$ & & 1024(0) & 992(0) \\
\hline & $850(0)$ & $819(0)$ & & 1071(2) & 1039(3) \\
\hline & $913(0)$ & $864(0)$ & & $1138(12)$ & $1106(18)$ \\
\hline & 1022(0) & 988(0) & & 1396(1) & 1357(2) \\
\hline & 1073(0) & $1040(0)$ & & $1456(0)$ & 1402(0) \\
\hline & 1273(0) & $1222(0)$ & & 3241(0) & $3162(0)$ \\
\hline & $1400(0)$ & $1360(0)$ & & 3252(0) & $3174(0)$ \\
\hline & $1455(0)$ & $1402(0)$ & & 3264(0) & $3185(0)$ \\
\hline & $3239(0)$ & $3162(0)$ & & & \\
\hline & $3254(0)$ & $3175(0)$ & & & \\
\hline
\end{tabular}


Table S2 Harmonic vibrational frequencies (in $\mathrm{cm}^{-1}$ ) and infrared intensities (in parentheses, in $\mathrm{km} / \mathrm{mol}$ ) for the structure Ib of $\mathrm{Cp}_{2} \mathrm{Fe}_{2}(\mathrm{NO})_{2}$ with $\mathrm{C}_{2 \mathrm{~h}}$ symmetry

\begin{tabular}{|c|c|c|c|c|c|}
\hline & B3LYP & BP86 & & B3LYP & BP86 \\
\hline \multirow{21}{*}{$a_{g}$} & $89(0)$ & $90(0)$ & \multirow[t]{15}{*}{$\mathrm{b}_{\mathrm{g}}$} & $63 \mathrm{i}(0)$ & $60 \mathrm{i}(0)$ \\
\hline & $164(0)$ & $156(0)$ & & $102(0)$ & $116(0)$ \\
\hline & $214(0)$ & $199(0)$ & & $321(0)$ & $312(0)$ \\
\hline & $317(0)$ & $319(0)$ & & $466(0)$ & $476(0)$ \\
\hline & $367(0)$ & $357(0)$ & & $571(0)$ & $550(0)$ \\
\hline & $559(0)$ & $546(0)$ & & $822(0)$ & 784(0) \\
\hline & $594(0)$ & $569(0)$ & & $841(0)$ & $811(0)$ \\
\hline & 717(0) & 707(0) & & $905(0)$ & $861(0)$ \\
\hline & $827(0)$ & 792(0) & & 1027(0) & $995(0)$ \\
\hline & $844(0)$ & $810(0)$ & & 1070(0) & $1038(0)$ \\
\hline & $859(0)$ & $824(0)$ & & 1276(0) & $1224(0)$ \\
\hline & $935(0)$ & $888(0)$ & & 1387(0) & 1349(0) \\
\hline & 1019(0) & $987(0)$ & & $1465(0)$ & 1412(0) \\
\hline & 1073(0) & 1042(0) & & $3238(0)$ & 3161(0) \\
\hline & 1142(0) & 1110(0) & & 3253(0) & $3178(0)$ \\
\hline & 1402(0) & 1366(0) & \multirow[t]{22}{*}{$\mathrm{b}_{\mathrm{u}}$} & $116(3)$ & $105(1)$ \\
\hline & $1455(0)$ & 1402(0) & & 147(3) & $142(5)$ \\
\hline & 1830(0) & $1769(0)$ & & $305(10)$ & 306(10) \\
\hline & 3244(0) & $3168(0)$ & & $349(1)$ & $347(0)$ \\
\hline & $3259(0)$ & 3182(0) & & 563(133) & 543(73) \\
\hline & $3285(0)$ & $3206(0)$ & & $592(5)$ & 571(24) \\
\hline \multirow[t]{16}{*}{$a_{u}$} & $8(2)$ & $18(2)$ & & $705(3)$ & $686(4)$ \\
\hline & $56 \mathrm{i}(0)$ & $52 \mathrm{i}(0)$ & & $824(135)$ & 788(89) \\
\hline & $85(0)$ & $85(0)$ & & $838(20)$ & 806(46) \\
\hline & $326(7)$ & $323(6)$ & & 851(37) & $816(18)$ \\
\hline & 509(13) & $486(10)$ & & $935(31)$ & $889(36)$ \\
\hline & $584(0)$ & $558(1)$ & & 1018(13) & 987(16) \\
\hline & $828(1)$ & 788(0) & & 1073(0) & 1041(0) \\
\hline & $843(1)$ & $813(1)$ & & 1142(5) & 1110(10) \\
\hline & $906(1)$ & $862(3)$ & & 1403(10) & 1366(6) \\
\hline & $1028(25)$ & 995(25) & & 1455(7) & 1402(7) \\
\hline & $1075(0)$ & 1041(1) & & 1797(1888) & 1751(1307) \\
\hline & 1276(0) & 1224(0) & & $3243(2)$ & $3168(3)$ \\
\hline & 1402(0) & 1359(0) & & 3258(1) & 3182(1) \\
\hline & $1465(5)$ & 1413(4) & & $3285(1)$ & $3206(2)$ \\
\hline & $3238(0)$ & 3161(0) & & & \\
\hline & $3253(1)$ & $3178(1)$ & & & \\
\hline
\end{tabular}


Table S3. Harmonic vibrational frequencies (in $\mathrm{cm}^{-1}$ ) and infrared intensities (in parentheses, in $\mathrm{km} / \mathrm{mol}$ ) for the structure IIa of $\mathrm{Cp}_{2} \mathrm{Fe}_{2}(\mu-\mathrm{NO})$ with $\mathrm{C}_{2 \mathrm{v}}$ symmetry

\begin{tabular}{|c|c|c|c|c|c|}
\hline & B3LYP & BP86 & & B3LYP & BP86 \\
\hline \multirow{19}{*}{$a_{1}$} & $63(0)$ & $71(1)$ & $b_{1}$ & $44(1)$ & $39(1)$ \\
\hline & $196(0)$ & 201(0) & & $56(0)$ & $66(0)$ \\
\hline & $317(4)$ & $336(9)$ & & $309(4)$ & $306(5)$ \\
\hline & $441(0)$ & $457(1)$ & & $439(3)$ & $435(2)$ \\
\hline & $555(3)$ & $552(0)$ & & $586(2)$ & $570(1)$ \\
\hline & $584(1)$ & $591(2)$ & & $814(0)$ & $773(0)$ \\
\hline & $795(2)$ & $765(0)$ & & $827(0)$ & $801(0)$ \\
\hline & 804(14) & $772(6)$ & & $884(2)$ & $836(1)$ \\
\hline & $842(0)$ & $812(0)$ & & $1018(29)$ & 982(31) \\
\hline & $897(2)$ & $852(3)$ & & $1066(0)$ & $1039(0)$ \\
\hline & $1018(25)$ & $985(26)$ & & 1271(0) & $1220(0)$ \\
\hline & $1068(1)$ & 1034(1) & & 1389(2) & 1353(1) \\
\hline & 1132(1) & $1100(0)$ & & $1454(6)$ & $1400(4)$ \\
\hline & $1384(0)$ & 1341(0) & & $3238(0)$ & $3161(0)$ \\
\hline & $1458(3)$ & $1405(3)$ & & $3250(1)$ & 3173(2) \\
\hline & 1703(619) & $1647(497)$ & $\mathrm{b}_{2}$ & $154(1)$ & $38(6)$ \\
\hline & $3235(0)$ & $3161(0)$ & & $255(0)$ & $206(1)$ \\
\hline & 3253(0) & $3176(1)$ & & $311 \mathrm{i}(84)$ & $341(0)$ \\
\hline & $3263(0)$ & $3186(0)$ & & $312(9)$ & $357(6)$ \\
\hline \multirow[t]{14}{*}{$\mathrm{a}_{2}$} & $27(0)$ & $39(0)$ & & 470(9) & $547(2)$ \\
\hline & $159(0)$ & $79(0)$ & & $574(2)$ & 721(83) \\
\hline & $345(0)$ & $325(0)$ & & 790(79) & 763(85) \\
\hline & $584(0)$ & $564(0)$ & & 795(71) & 799(96) \\
\hline & $816(0)$ & 768(0) & & $835(0)$ & $830(173)$ \\
\hline & $830(0)$ & 798(0) & & $895(0)$ & $888(312)$ \\
\hline & $879(0)$ & $833(0)$ & & 1016(0) & $985(10)$ \\
\hline & 1018(0) & $984(0)$ & & $1063(0)$ & $1048(73)$ \\
\hline & 1070(0) & 1038(0) & & $1133(12)$ & $1122(322)$ \\
\hline & 1271(0) & $1220(0)$ & & $1377(1)$ & 1334(1) \\
\hline & 1392(0) & 1354(0) & & 1456(0) & $1411(68)$ \\
\hline & 1453(0) & $1400(0)$ & & $3235(0)$ & $3161(5)$ \\
\hline & $3237(0)$ & 3161(0) & & $3253(1)$ & $3176(8)$ \\
\hline & $3250(0)$ & $3173(0)$ & & $3263(0)$ & $3187(2)$ \\
\hline
\end{tabular}


Table S4 Harmonic vibrational frequencies (in $\mathrm{cm}^{-1}$ ) and infrared intensities (in parentheses, in $\mathrm{km} / \mathrm{mol}$ ) for the structure Id of $\mathrm{Cp}_{2} \mathrm{Fe}_{2} \mathrm{NO}$ with $\mathrm{C}_{\mathrm{s}}$ symmetry

\begin{tabular}{|c|c|c|c|c|c|}
\hline & B3LYP & BP86 & & B3LYP & BP86 \\
\hline \multirow[t]{37}{*}{$a_{-}$} & $51(0)$ & $53(3)$ & a" & $38 \mathrm{i}(0)$ & $81 \mathrm{i}(2)$ \\
\hline & $67(1)$ & $65(0)$ & & $43(0)$ & $69(0)$ \\
\hline & 101(1) & $152(0)$ & & $79(0)$ & $78(0)$ \\
\hline & $162(2)$ & $211(26)$ & & $97(0)$ & $146(1)$ \\
\hline & $252(0)$ & $285(22)$ & & $199(0)$ & $268(1)$ \\
\hline & $295(0)$ & $307(2)$ & & $213(2)$ & $315(2)$ \\
\hline & $313(2)$ & $333(2)$ & & $325(0)$ & $440(5)$ \\
\hline & $347(4)$ & $416(0)$ & & $580(0)$ & $546(0)$ \\
\hline & $371(11)$ & $487(8)$ & & $606(0)$ & $564(1)$ \\
\hline & $510(26)$ & $554(1)$ & & $744(2)$ & $710(2)$ \\
\hline & $597(3)$ & $559(1)$ & & $777(0)$ & $767(0)$ \\
\hline & $752(12)$ & $712(16)$ & & $815(0)$ & $778(0)$ \\
\hline & 767(83) & 758(39) & & $836(0)$ & 799(0) \\
\hline & $637(0)$ & $694(8)$ & & $871(0)$ & $826(0)$ \\
\hline & $795(73)$ & 775(19) & & 880(0) & $842(0)$ \\
\hline & $820(17)$ & $789(3)$ & & $1001(21)$ & 951(13) \\
\hline & $830(1)$ & $795(3)$ & & $1025(20)$ & $980(15)$ \\
\hline & $838(5)$ & $800(17)$ & & $1057(0)$ & $1037(0)$ \\
\hline & $859(0)$ & $842(6)$ & & $1068(0)$ & 1039(1) \\
\hline & $903(4)$ & $851(2)$ & & $1262(0)$ & $1204(0)$ \\
\hline & $1002(7)$ & $962(7)$ & & $1272(0)$ & $1219(0)$ \\
\hline & $1009(14)$ & 984(11) & & 1377(1) & 1313(3) \\
\hline & 1061(4) & $1008(5)$ & & $1389(0)$ & $1356(1)$ \\
\hline & 1064(2) & $1030(1)$ & & $1430(0)$ & $1386(0)$ \\
\hline & 1098(4) & $1066(16)$ & & $1465(0)$ & 1393(3) \\
\hline & $1134(2)$ & $1099(12)$ & & $3220(0)$ & $3152(1)$ \\
\hline & 1364(7) & $1296(5)$ & & $3223(1)$ & $3145(0)$ \\
\hline & $1385(16)$ & $1343(2)$ & & $3243(2)$ & $3168(1)$ \\
\hline & $1434(2)$ & $1384(1)$ & & $3244(0)$ & $3172(1)$ \\
\hline & $1450(4)$ & 1398(2) & & & \\
\hline & 1789(538) & $1756(865)$ & & & \\
\hline & $3169(0)$ & $3153(1)$ & & & \\
\hline & $3230(1)$ & $3158(1)$ & & & \\
\hline & $3231(0)$ & $3161(0)$ & & & \\
\hline & $3243(3)$ & $3170(1)$ & & & \\
\hline & $3250(1)$ & $3180(0)$ & & & \\
\hline & $3258(1)$ & $3184(0)$ & & & \\
\hline
\end{tabular}


Table S5. Harmonic vibrational frequencies (in $\mathrm{cm}^{-1}$ ) and infrared intensities (in parentheses, in $\mathrm{km} / \mathrm{mol}$ ) for the structure III of $\mathrm{Cp}_{2} \mathrm{Co}_{2}(\mu-\mathrm{NO})_{2}$ with $\mathrm{C}_{2 \mathrm{v}}$ symmetry

\begin{tabular}{|c|c|c|c|c|c|}
\hline & B3LYP & BP86 & & B3LYP & BP86 \\
\hline \multirow[t]{21}{*}{$a_{1}$} & $42(1)$ & $41(1)$ & $b_{1}$ & $13 \mathrm{i}(0)$ & $8(0)$ \\
\hline & $116(1)$ & $111(0)$ & & $04(0)$ & 103(1) \\
\hline & $198(0)$ & 201(0) & & $330(7)$ & $322(7)$ \\
\hline & 299(3) & 291(5) & & $429(10)$ & $409(6)$ \\
\hline & 411(4) & $406(1)$ & & $533(3)$ & $530(3)$ \\
\hline & $517(6)$ & $510(6)$ & & $592(2)$ & $570(2)$ \\
\hline & $572(1)$ & $546(1)$ & & $831(9)$ & $798(10)$ \\
\hline & $597(4)$ & $567(5)$ & & 843(0) & $812(0)$ \\
\hline & $808(9)$ & $768(7)$ & & $903(0)$ & $855(0)$ \\
\hline & $830(0)$ & 791(1) & & 1014(23) & $980(21)$ \\
\hline & $844(2)$ & $810(1)$ & & $1068(0)$ & $1036(0)$ \\
\hline & $901(0)$ & $853(0)$ & & 1271(0) & $1221(0)$ \\
\hline & $1025(23)$ & 995(23) & & 1387(0) & $1347(0)$ \\
\hline & $1067(2)$ & 1035(1) & & $1451(4)$ & 1397(6) \\
\hline & $1135(2)$ & 1104(2) & & $1643(845)$ & $1588(685)$ \\
\hline & 1392(1) & 1352(0) & & $3240(0)$ & $3163(0)$ \\
\hline & $1460(3)$ & $1411(2)$ & & 3253(0) & $3175(2)$ \\
\hline & 1689(199) & $1628(145)$ & $\mathrm{b}_{2}$ & $140(2)$ & 139(1) \\
\hline & $3238(0)$ & $3162(0)$ & & $227(4)$ & $228(3)$ \\
\hline & $3253(1)$ & $3177(3)$ & & $333(19)$ & $314(8)$ \\
\hline & $3264(0)$ & $3187(0)$ & & $354(8)$ & $348(3)$ \\
\hline \multirow{16}{*}{$a_{2}$} & $20 \mathrm{i}(0)$ & $22 \mathrm{i}(0)$ & & $584(2)$ & $555(2)$ \\
\hline & $129(0)$ & $124(0)$ & & $796(249)$ & $759(131)$ \\
\hline & $272(0)$ & $267(0)$ & & $817(18)$ & $785(44)$ \\
\hline & $365(0)$ & $354(0)$ & & $828(77)$ & 792(197) \\
\hline & $573(0)$ & $531(0)$ & & $842(4)$ & $808(10)$ \\
\hline & $597(0)$ & $579(0)$ & & $899(2)$ & $851(3)$ \\
\hline & $830(0)$ & 797(0) & & 1024(1) & $995(0)$ \\
\hline & $841(0)$ & $807(0)$ & & 1067(0) & 1034(0) \\
\hline & $903(0)$ & $855(0)$ & & 1136(6) & 1104(7) \\
\hline & 1014(0) & $979(0)$ & & 1391(0) & 1349(1) \\
\hline & 1067(0) & 1035(0) & & $1460(1)$ & 1411(1) \\
\hline & 1271(0) & 1221(0) & & $3238(0)$ & $3162(0)$ \\
\hline & 1390(0) & 1349(0) & & $3253(0)$ & 3177(0) \\
\hline & $1450(0)$ & 1396(0) & & $3264(0)$ & $3187(2)$ \\
\hline & $3240(0)$ & 3163(0) & & & \\
\hline & $3253(0)$ & $3175(0)$ & & & \\
\hline
\end{tabular}


Table S6. Harmonic vibrational frequencies (in $\mathrm{cm}^{-1}$ ) and infrared intensities (in parentheses, in $\mathrm{km} / \mathrm{mol})$ for the structure IVa of $\mathrm{Cp}_{2} \mathrm{Co}_{2}(\mu-\mathrm{NO})$ with $\mathrm{C}_{2 \mathrm{v}}$ symmetry

\begin{tabular}{|c|c|c|c|c|c|}
\hline & B3LYP & BP86 & & B3LYP & BP86 \\
\hline \multirow{19}{*}{$a_{1}$} & $60(1)$ & $63(1)$ & \multirow{15}{*}{$\mathrm{b}_{1}$} & $8 \mathrm{i}(0)$ & $22(1)$ \\
\hline & 191(0) & $193(0)$ & & $55(0)$ & $57(0)$ \\
\hline & $304(6)$ & $320(10)$ & & $311(4)$ & $323(5)$ \\
\hline & $408(2)$ & $405(0)$ & & $482(6)$ & $449(5)$ \\
\hline & $571(0)$ & $551(0)$ & & $590(1)$ & $563(1)$ \\
\hline & $583(0)$ & $562(0)$ & & $827(0)$ & $789(0)$ \\
\hline & 790(1) & $755(4)$ & & $836(0)$ & $799(0)$ \\
\hline & 801(24) & 770(12) & & $888(0)$ & $841(0)$ \\
\hline & $842(1)$ & $810(0)$ & & $1010(30)$ & $976(29)$ \\
\hline & $897(2)$ & $851(3)$ & & $1068(0)$ & 1037(0) \\
\hline & $1027(23)$ & 994(24) & & 1271(0) & 1220(0) \\
\hline & $1066(0)$ & 1035(0) & & 1385(1) & $1345(0)$ \\
\hline & 1134(1) & 1102(1) & & 1446(7) & 1394(8) \\
\hline & $1388(2)$ & 1348(0) & & $3237(1)$ & $3160(1)$ \\
\hline & $1465(2)$ & $1412(2)$ & & $3248(2)$ & 3171(4) \\
\hline & 1678(699) & $1623(519)$ & \multirow[t]{18}{*}{$\mathrm{b}_{2}$} & $162(1)$ & $53(2)$ \\
\hline & $3235(0)$ & $3159(0)$ & & $269(0)$ & $222(5)$ \\
\hline & $3254(1)$ & $3177(2)$ & & $319(1)$ & $265(0)$ \\
\hline & $3263(0)$ & $3186(0)$ & & $423(14)$ & $347(3)$ \\
\hline \multirow[t]{14}{*}{$a_{2}$} & $20 \mathrm{i}(0)$ & $11 \mathrm{i}(0)$ & & $533 \mathrm{i}(24)$ & $540(2)$ \\
\hline & $122(0)$ & $126(0)$ & & $575(4)$ & $572(10)$ \\
\hline & $361(0)$ & $365(0)$ & & 0786(0) & $751(1)$ \\
\hline & $583(0)$ & $559(0)$ & & 795(97) & 766(83) \\
\hline & $826(0)$ & $782(0)$ & & 839(27) & $806(17)$ \\
\hline & $838(0)$ & 799(0) & & $898(0)$ & $851(0)$ \\
\hline & $887(0)$ & $837(0)$ & & $1025(4)$ & $993(2)$ \\
\hline & 1009(0) & $976(0)$ & & $1062(10)$ & $1027(5)$ \\
\hline & 1066(0) & 1036(0) & & $1134(10)$ & $1102(14)$ \\
\hline & 1271(0) & 1220(0) & & $1385(37)$ & $1339(18)$ \\
\hline & 1386(0) & 1346(0) & & $1463(1)$ & 1411(0) \\
\hline & $1445(0)$ & 1392(0) & & $3235(0)$ & 3159(0) \\
\hline & $3237(0)$ & 3159(0) & & $3253(1)$ & $3177(0)$ \\
\hline & $3248(0)$ & 3171(0) & & $3263(1)$ & $3186(2)$ \\
\hline
\end{tabular}


Table S7. Harmonic vibrational frequencies (in $\mathrm{cm}^{-1}$ ) and infrared intensities (in parentheses, in $\mathrm{km} / \mathrm{mol}$ ) for the structure $\mathbf{I V b}$ of $\mathrm{CpCo}_{2}(\mu-\mathrm{Cp})(\mathrm{NO})$ with $\mathrm{C}_{\mathrm{s}}$ symmetry

\begin{tabular}{|c|c|c|c|c|c|}
\hline & B3LYP & BP86 & & B3LYP & BP86 \\
\hline \multirow[t]{37}{*}{$a^{\prime}$} & $34(2)$ & 73(0) & a" & $12(0)$ & $26 \mathrm{i}(0)$ \\
\hline & $67(3)$ & $103(2)$ & & $32(0)$ & $38(0)$ \\
\hline & $110(18)$ & $181(5)$ & & $52(0)$ & $74(0)$ \\
\hline & $146(5)$ & 195(1) & & $93(0)$ & $103(0)$ \\
\hline & 231(15) & $295(7)$ & & $216(2)$ & 307(4) \\
\hline & 251(5) & $355(2)$ & & $243(0)$ & $329(3)$ \\
\hline & $298(2)$ & 383(13) & & $354(2)$ & $466(6)$ \\
\hline & $338(4)$ & $408(5)$ & & $583(0)$ & $512(2)$ \\
\hline & $356(4)$ & $556(3)$ & & $597(0)$ & $559(0)$ \\
\hline & 516(17) & $520(69)$ & & 774(0) & 741(1) \\
\hline & 594(14) & $596(7)$ & & $778(0)$ & 744(1) \\
\hline & $607(0)$ & $666(3)$ & & $827(0)$ & 770(0) \\
\hline & 787(88) & $759(58)$ & & $840(0)$ & $795(0)$ \\
\hline & $796(65)$ & 769(49) & & $875(0)$ & $836(0)$ \\
\hline & 801(20) & 793(1) & & $897(0)$ & $841(0)$ \\
\hline & $837(24)$ & 798(4) & & $958(6)$ & $875(4)$ \\
\hline & 840(20) & $812(3)$ & & 1021(19) & 1014(12) \\
\hline & $846(12)$ & $817(9)$ & & $1055(10)$ & $1025(0)$ \\
\hline & 883(8) & $859(8)$ & & 1061(1) & 993(18) \\
\hline & $906(35)$ & $960(12)$ & & 1248(0) & $1185(0)$ \\
\hline & 1003(18) & $968(2)$ & & 1269(0) & 1217(0) \\
\hline & 1007(9) & $1028(1)$ & & 1362(0) & $1306(0)$ \\
\hline & 1061(20) & 1033(1) & & 1364(0) & $1315(0)$ \\
\hline & $1069(0)$ & 1082(23) & & 1453(2) & 1389(1) \\
\hline & 1124(2) & $1095(7)$ & & 1464(0) & $1416(0)$ \\
\hline & $1128(4)$ & 1116(29) & & $3225(0)$ & 3152(1) \\
\hline & 1383(3) & $1337(0)$ & & $3237(0)$ & 3163(0) \\
\hline & 1383(80) & 1346(13) & & $3242(2)$ & $3175(1)$ \\
\hline & $1439(11)$ & 1376(4) & & $3255(0)$ & 3184(0) \\
\hline & $1440(1)$ & 1377(4) & & & \\
\hline & 1816(1007) & $1783(743)$ & & & \\
\hline & $2920(17)$ & 2564(1) & & & \\
\hline & $3231(1)$ & $3160(0)$ & & & \\
\hline & $3245(0)$ & $3173(1)$ & & & \\
\hline & $3246(2)$ & $3167(2)$ & & & \\
\hline & $3260(2)$ & $3183(1)$ & & & \\
\hline & $3261(0)$ & $3189(2)$ & & & \\
\hline
\end{tabular}


Table S8. Harmonic vibrational frequencies (in $\mathrm{cm}^{-1}$ ) and infrared intensities (in parentheses, in $\mathrm{km} / \mathrm{mol}$ ) for the structure $\mathbf{V}$ of $\mathrm{Cp}_{2} \mathrm{Ni}_{2}(\mu-\mathrm{NO})_{2}$ with $\mathrm{C}_{1}$ symmetry

\begin{tabular}{|c|c|c|c|}
\hline B3LYP & BP86 & B3LYP & BP86 \\
\hline $57 i(0)$ & $19 \mathrm{i}(0)$ & $889(2)$ & $847(1)$ \\
\hline $21(0)$ & $13 \mathrm{i}(0)$ & 891(1) & $849(1)$ \\
\hline $27(0)$ & $46(0)$ & $892(0)$ & $850(2)$ \\
\hline $43(0)$ & $76(0)$ & $896(0)$ & $854(0)$ \\
\hline $94(0)$ & $101(0)$ & $1012(2)$ & $982(4)$ \\
\hline $119(0)$ & $122(1)$ & $1012(24)$ & 984(24) \\
\hline $120(1)$ & $126(0)$ & $1024(1)$ & $992(0)$ \\
\hline $147(0)$ & $161(0)$ & $1024(24)$ & 992(23) \\
\hline $183(0)$ & $189(0)$ & $1062(1)$ & $1032(0)$ \\
\hline $211(0)$ & $207(0)$ & 1064(0) & 1033(0) \\
\hline $222(0)$ & $223(0)$ & 1064(0) & 1034(2) \\
\hline $227(1)$ & $229(2)$ & 1064(1) & $1035(0)$ \\
\hline $262(2)$ & $247(2)$ & $1135(1)$ & $1105(1)$ \\
\hline $288(1)$ & 271(2) & $1135(0)$ & $1105(1)$ \\
\hline $311(9)$ & $284(2)$ & $1272(0)$ & $1221(0)$ \\
\hline $321(38)$ & $321(15)$ & $1272(0)$ & $1221(0)$ \\
\hline $356(0)$ & $331(5)$ & 1376(5) & $1337(0)$ \\
\hline $375(4)$ & $373(2)$ & 1377(1) & $1340(0)$ \\
\hline $428(0)$ & $420(5)$ & 1378(1) & 1341(1) \\
\hline $448(8)$ & $468(2)$ & 1379(3) & 1343(5) \\
\hline $571(4)$ & $556(0)$ & 1448(2) & 1398(2) \\
\hline $577(0)$ & $560(0)$ & 1449(4) & 1399(3) \\
\hline $581(3)$ & $561(1)$ & $1463(0)$ & 1411(0) \\
\hline $586(1)$ & $561(0)$ & 1463(6) & 1411(5) \\
\hline $587(0)$ & $579(2)$ & $1595(784)$ & $1536(677)$ \\
\hline $695(121)$ & 634(109) & $1696(452)$ & $1603(303)$ \\
\hline 782(94) & $759(68)$ & $3236(0)$ & $3162(0)$ \\
\hline $788(15)$ & 762(17) & $3236(0)$ & $3162(0)$ \\
\hline $800(70)$ & $768(50)$ & $3238(0)$ & $3163(0)$ \\
\hline 801(18) & 771(23) & $3238(0)$ & $3163(0)$ \\
\hline $813(18)$ & 778(22) & 3249(0) & $3175(0)$ \\
\hline $817(3)$ & 782(1) & $3250(1)$ & $3176(2)$ \\
\hline $836(0)$ & $806(0)$ & $3253(0)$ & $3178(0)$ \\
\hline $836(0)$ & $809(1)$ & $3253(1)$ & $3178(1)$ \\
\hline $838(4)$ & $810(0)$ & $3263(2)$ & $3188(2)$ \\
\hline $840(2)$ & $811(4)$ & $3263(0)$ & $3188(1)$ \\
\hline
\end{tabular}


Table S9. Harmonic vibrational frequencies (in $\mathrm{cm}^{-1}$ ) and infrared intensities (in parentheses, in $\mathrm{km} / \mathrm{mol}$ ) for the structure $\mathrm{VI}$ of $\mathrm{Cp}_{2} \mathrm{Ni}_{2}(\mu-\mathrm{NO})$ with $\mathrm{C}_{2 \mathrm{v}}$ symmetry

\begin{tabular}{|c|c|c|c|c|c|}
\hline & B3LYP & BP86 & & B3LYP & BP86 \\
\hline \multirow[t]{19}{*}{$a_{1}$} & $53(0)$ & $58(0)$ & $b_{1}$ & $7 \mathrm{i}(0)$ & $4 \mathrm{i}(0)$ \\
\hline & $175(0)$ & $188(0)$ & & $59(0)$ & $62(0)$ \\
\hline & $277(2)$ & $285(3)$ & & $269(2)$ & $277(3)$ \\
\hline & $366(1)$ & $373(0)$ & & $441(3)$ & $441(4)$ \\
\hline & $521(1)$ & $550(1)$ & & $596(0)$ & $564(0)$ \\
\hline & $596(0)$ & $568(0)$ & & $801(0)$ & $771(0)$ \\
\hline & $794(4)$ & $761(1)$ & & $844(0)$ & $807(0)$ \\
\hline & 798(38) & $766(32)$ & & $884(0)$ & $840(0)$ \\
\hline & $845(0)$ & $812(0)$ & & 1014(38) & $979(34)$ \\
\hline & $888(0)$ & $844(0)$ & & $1065(0)$ & $1032(0)$ \\
\hline & $1020(27)$ & $991(25)$ & & $1270(0)$ & $1219(0)$ \\
\hline & $1066(0)$ & $1035(0)$ & & $1379(0)$ & $1336(0)$ \\
\hline & $1133(1)$ & $1102(2)$ & & $1451(2)$ & 1394(4) \\
\hline & $1383(0)$ & $1347(2)$ & & $3235(0)$ & $3161(0)$ \\
\hline & $1458(1)$ & $1410(1)$ & & $3248(3)$ & $3174(5)$ \\
\hline & $1678(575)$ & $1627(526)$ & $\mathrm{b}_{2}$ & $101(0)$ & $102(0)$ \\
\hline & $3235(0)$ & $3161(0)$ & & $223(0)$ & $233(0)$ \\
\hline & $3251(2)$ & $3177(3)$ & & $269(11)$ & $282(1)$ \\
\hline & $3262(0)$ & $3187(1)$ & & $347(44)$ & $351(16)$ \\
\hline \multirow[t]{14}{*}{$\mathrm{a}_{2}$} & $13 \mathrm{i}(0)$ & $13 \mathrm{i}(0)$ & & $574(56)$ & $555(1)$ \\
\hline & $112(0)$ & $112(0)$ & & 612(47) & $638(56)$ \\
\hline & $281(0)$ & 291(0) & & $789(22)$ & 754(14) \\
\hline & $594(0)$ & $564(0)$ & & $794(145)$ & 764(98) \\
\hline & 798(0) & 767(0) & & $844(0)$ & $809(16)$ \\
\hline & $842(0)$ & $806(0)$ & & $887(0)$ & $843(0)$ \\
\hline & $880(0)$ & $835(0)$ & & 1019(4) & $990(5)$ \\
\hline & 1014(0) & $978(0)$ & & 1063(1) & $1030(10)$ \\
\hline & 1064(0) & 1032(0) & & $1133(1)$ & 1102(4) \\
\hline & $1270(0)$ & $1219(0)$ & & $1382(1)$ & $1340(26)$ \\
\hline & $1380(0)$ & $1337(0)$ & & 1457(3) & 1409(3) \\
\hline & $1450(0)$ & 1393(0) & & $3235(0)$ & $3161(0)$ \\
\hline & $3234(0)$ & $3161(0)$ & & $3250(1)$ & 3176(1) \\
\hline & $3248(0)$ & $3173(0)$ & & $3261(2)$ & $3187(3)$ \\
\hline
\end{tabular}


Table S10. Harmonic vibrational frequencies (in $\mathrm{cm}^{-1}$ ) and infrared intensities (in parentheses, in $\mathrm{km} / \mathrm{mol}$ ) for the structure VIIa of $\mathrm{Cp}_{2} \mathrm{Ni}_{2}(\mu-\mathrm{CO})_{2}$ with $\mathrm{C}_{2 \mathrm{~h}}$ symmetry

\begin{tabular}{|c|c|c|c|c|c|}
\hline$a_{g}$ & $111(0)$ & $120(0)$ & $b_{g}$ & $19 \mathrm{i}(0)$ & $21 \mathrm{i}(0)$ \\
\hline \multirow{36}{*}{$a_{u}$} & $174(0)$ & $180(0)$ & \multirow{36}{*}{$\mathrm{b}_{\mathrm{u}}$} & $129(0)$ & $132(0)$ \\
\hline & $248(0)$ & $251(0)$ & & $235(0)$ & $239(0)$ \\
\hline & $298(0)$ & $311(0)$ & & $410(0)$ & $407(0)$ \\
\hline & $383(0)$ & $383(0)$ & & $597(0)$ & $573(0)$ \\
\hline & $439(0)$ & $458(0)$ & & 775(0) & 743(0) \\
\hline & $459(0)$ & $468(0)$ & & $843(0)$ & $813(0)$ \\
\hline & $597(0)$ & $573(0)$ & & $898(0)$ & $857(0)$ \\
\hline & 797(0) & $768(0)$ & & 1031(0) & $1002(0)$ \\
\hline & $844(0)$ & $813(0)$ & & $1068(0)$ & $1037(0)$ \\
\hline & $847(0)$ & $818(0)$ & & 1272(0) & 1222(0) \\
\hline & $908(0)$ & $869(0)$ & & 1381(0) & 1342(0) \\
\hline & 1001(0) & $971(0)$ & & $1474(0)$ & $1425(0)$ \\
\hline & 1066(0) & 1036(0) & & $3238(0)$ & $3165(0)$ \\
\hline & 1134(0) & 1104(0) & & $3255(0)$ & 3182(0) \\
\hline & 1390(0) & $1350(0)$ & & $97(0)$ & $99(1)$ \\
\hline & $1438(0)$ & $1389(0)$ & & $222(4)$ & $221(3)$ \\
\hline & 1963(0) & $1870(0)$ & & 276(13) & $276(12)$ \\
\hline & $3238(0)$ & $3164(0)$ & & $328(60)$ & $330(30)$ \\
\hline & $3249(0)$ & $3176(0)$ & & 474(22) & $486(13)$ \\
\hline & $3264(0)$ & $3190(0)$ & & $588(18)$ & $564(9)$ \\
\hline & $16 \mathrm{i}(0)$ & $18 \mathrm{i}(0)$ & & $670(416)$ & $662(349)$ \\
\hline & $28 \mathrm{i}(1)$ & $30 \mathrm{i}(1)$ & & $794(211)$ & 766(199) \\
\hline & $92(0)$ & $91(0)$ & & $840(13)$ & 809(3) \\
\hline & $228(1)$ & $231(1)$ & & $846(10)$ & $816(12)$ \\
\hline & $470(11)$ & 471(11) & & $906(7)$ & $868(9)$ \\
\hline & $577(0)$ & $555(0)$ & & $1001(22)$ & 971(20) \\
\hline & $775(1)$ & 743(1) & & $1063(12)$ & 1032(8) \\
\hline & $833(0)$ & $803(0)$ & & $1133(0)$ & $1104(0)$ \\
\hline & 897(0) & $856(0)$ & & $1384(47)$ & $1344(38)$ \\
\hline & 1031(39) & $1002(37)$ & & $1437(10)$ & $1388(12)$ \\
\hline & $1063(0)$ & $1033(0)$ & & $1908(1082)$ & $1830(850)$ \\
\hline & $1272(0)$ & $1222(0)$ & & $3238(1)$ & $3164(0)$ \\
\hline & $1374(1)$ & 1334(1) & & $3249(1)$ & $3176(1)$ \\
\hline & $1473(1)$ & $1424(1)$ & & $3264(3)$ & $3190(4)$ \\
\hline & $3238(0)$ & $3165(0)$ & & & \\
\hline & $3255(1)$ & 3182(1) & & & \\
\hline
\end{tabular}


Table S11. Harmonic vibrational frequencies $\left(\right.$ in $\mathrm{cm}^{-1}$ ) and infrared intensities (in parentheses, in $\mathrm{km} / \mathrm{mol})$ for the structure VIIb of $\mathrm{Cp}_{2} \mathrm{Ni}_{2}(\mu-\mathrm{CO})_{2}$ with $\mathrm{C}_{2 \mathrm{~h}}$ symmetry

\begin{tabular}{|c|c|c|c|c|c|}
\hline & B3LYP & BP86 & & B3LYP & BP86 \\
\hline \multirow[t]{21}{*}{$a_{g}$} & $78(0)$ & $95(0)$ & $b_{g}$ & $9 \mathrm{i}(0)$ & $9 \mathrm{i}(0)$ \\
\hline & $159(0)$ & $156(0)$ & & $133(0)$ & $132(0)$ \\
\hline & $179(0)$ & $169(0)$ & & $245(0)$ & $237(0)$ \\
\hline & $237(0)$ & $230(0)$ & & $279(0)$ & $244(0)$ \\
\hline & $342(0)$ & $349(0)$ & & $584(0)$ & $559(0)$ \\
\hline & $375(0)$ & $395(0)$ & & $802(0)$ & 772(0) \\
\hline & $418(0)$ & $430(0)$ & & $837(0)$ & $805(0)$ \\
\hline & $597(0)$ & $568(0)$ & & $891(0)$ & $848(0)$ \\
\hline & 796(0) & 763(0) & & $1018(0)$ & $987(0)$ \\
\hline & 798(0) & 767(0) & & 1064(0) & 1032(0) \\
\hline & $846(0)$ & $817(0)$ & & $1272(0)$ & $1223(0)$ \\
\hline & $895(0)$ & $853(0)$ & & $1375(0)$ & 1331(0) \\
\hline & 1019(0) & 990(0) & & $1455(0)$ & $1403(0)$ \\
\hline & $1066(0)$ & $1037(0)$ & & $3235(0)$ & $3163(0)$ \\
\hline & $1136(0)$ & $1106(0)$ & & $3249(0)$ & $3175(0)$ \\
\hline & $1380(0)$ & $1345(0)$ & $\mathrm{bu}$ & $85(1)$ & $87(1)$ \\
\hline & $1456(0)$ & $1408(0)$ & & $173(1)$ & $175(0)$ \\
\hline & 1977(0) & $1876(0)$ & & 211(5) & 201(5) \\
\hline & $3235(0)$ & $3162(0)$ & & $312(23)$ & $305(17)$ \\
\hline & $3251(0)$ & $3179(0)$ & & $327(31)$ & $387(15)$ \\
\hline & $3262(0)$ & $3189(0)$ & & $365(24)$ & $454(16)$ \\
\hline \multirow[t]{16}{*}{$\mathrm{au}$} & $7(0)$ & $13(0)$ & & $587(0)$ & $558(1)$ \\
\hline & $25(0)$ & $24(1)$ & & 793(219) & $761(162)$ \\
\hline & $87(0)$ & $81(0)$ & & $798(13)$ & $766(34)$ \\
\hline & $235(1)$ & $228(1)$ & & $841(8)$ & $809(16)$ \\
\hline & $395(6)$ & $367(3)$ & & 892(4) & $850(9)$ \\
\hline & $598(0)$ & $568(0)$ & & $1019(26)$ & $990(26)$ \\
\hline & $801(0)$ & $771(0)$ & & 1064(1) & 1032(3) \\
\hline & $846(0)$ & $815(0)$ & & $1135(0)$ & 1106(1) \\
\hline & 892(1) & $850(1)$ & & $1376(1)$ & $1335(7)$ \\
\hline & $1018(36)$ & 987(33) & & $1456(2)$ & $1408(2)$ \\
\hline & $1067(0)$ & $1038(0)$ & & 1916(1218) & $1834(922)$ \\
\hline & $1272(0)$ & $1223(0)$ & & $3235(0)$ & $3162(0)$ \\
\hline & $1380(0)$ & $1345(0)$ & & $3251(1)$ & $3179(1)$ \\
\hline & $1455(3)$ & 1403(4) & & $3262(2)$ & $3189(3)$ \\
\hline & $3235(0)$ & $3163(0)$ & & & \\
\hline & $3249(2)$ & $3175(3)$ & & & \\
\hline
\end{tabular}


Table S12 Harmonic vibrational frequencies (in $\mathrm{cm}^{-1}$ ) and infrared intensities (in parentheses, in $\mathrm{km} / \mathrm{mol}$ ) for the structure VIIc of triplet $\perp-\mathrm{Cp}_{2} \mathrm{Ni}_{2}(\mathrm{CO})_{2}$ with $\mathrm{C}_{2 \mathrm{~h}}$ symmetry

\begin{tabular}{|c|c|c|c|c|c|}
\hline & B3LYP & BP86 & & B3LYP & BP86 \\
\hline \multirow[t]{21}{*}{$a_{g}$} & $57(0)$ & $74(0)$ & $b_{g}$ & $27 \mathrm{i}(0)$ & $35(0)$ \\
\hline & $109(0)$ & $111(0)$ & & $46(0)$ & $65(0)$ \\
\hline & $131(0)$ & $127(0)$ & & $183(0)$ & $129(0)$ \\
\hline & $236(0)$ & $242(0)$ & & $366(0)$ & $252(0)$ \\
\hline & $325(0)$ & $342(0)$ & & $674(0)$ & $574(0)$ \\
\hline & $360(0)$ & $381(0)$ & & $761(0)$ & $694(0)$ \\
\hline & $378(0)$ & $425(0)$ & & $836(0)$ & $780(0)$ \\
\hline & $622(0)$ & $607(0)$ & & $972(0)$ & $815(0)$ \\
\hline & $750(0)$ & $717(0)$ & & $1015(0)$ & $971(0)$ \\
\hline & $793(0)$ & $764(0)$ & & $1145(0)$ & $1027(0)$ \\
\hline & $830(0)$ & $800(0)$ & & $1266(0)$ & $1212(0)$ \\
\hline & $902(0)$ & $872(0)$ & & $1429(0)$ & $1326(0)$ \\
\hline & 1014(0) & $984(0)$ & & $3210(0)$ & $1375(0)$ \\
\hline & 1064(0) & 1034(0) & & $3233(0)$ & $3139(0)$ \\
\hline & $1115(0)$ & $1078(0)$ & & $8322(0)$ & $3161(0)$ \\
\hline & $1372(0)$ & $1334(0)$ & $\mathrm{bu}$ & $67(8)$ & $56(1)$ \\
\hline & $1447(0)$ & $1395(0)$ & & $127(17)$ & $147(10)$ \\
\hline & 2078(0) & 1966(0) & & $196 \mathrm{i}(74)$ & $273(7)$ \\
\hline & $3219(0)$ & $3145(0)$ & & $280(5)$ & 287(4) \\
\hline & $3230(0)$ & $3154(0)$ & & 298(74) & $355(14)$ \\
\hline & $3246(0)$ & 3171(0) & & $357(3)$ & $416(23)$ \\
\hline \multirow[t]{16}{*}{ au } & $26(0)$ & $30 \mathrm{i}(0)$ & & $560(14)$ & $601(0)$ \\
\hline & $43 \mathrm{i}(0)$ & 21(1) & & $737(110)$ & 707(78) \\
\hline & $57(0)$ & $56(0)$ & & $770(31)$ & $756(68)$ \\
\hline & $157(0)$ & $177(0)$ & & $823(6)$ & 793(17) \\
\hline & $245(2)$ & 234(1) & & 881(44) & $865(12)$ \\
\hline & $606(0)$ & $578(0)$ & & $1012(18)$ & 980(19) \\
\hline & $746(3)$ & 719(4) & & 1037(3) & $1027(1)$ \\
\hline & $815(1)$ & $778(2)$ & & 1112(0) & 1077(0) \\
\hline & $852(1)$ & $821(1)$ & & 1303(10) & 1323(7) \\
\hline & $1007(36)$ & 974(36) & & 1448(1) & 1395(1) \\
\hline & 1061(0) & 1032(0) & & 2044(2940) & $1953(2223)$ \\
\hline & $1264(0)$ & $1212(0)$ & & 3218(1) & $3144(1)$ \\
\hline & 1382(1) & $1340(1)$ & & $3230(0)$ & 3154(0) \\
\hline & 1432(4) & 1378(5) & & $3246(4)$ & 3171(5) \\
\hline & $3214(0)$ & $3140(0)$ & & & \\
\hline & $3234(5)$ & $3161(4)$ & & & \\
\hline
\end{tabular}


Table S13. Harmonic vibrational frequencies (in $\mathrm{cm}^{-1}$ ) and infrared intensities (in parentheses, in $\mathrm{km} / \mathrm{mol})$ for the structure VIIIa of singlet $\mathrm{Cp}_{2} \mathrm{Ni}_{2}(\mu-\mathrm{CO})$ with $\mathrm{C}_{2 \mathrm{v}}$ symmetry

\begin{tabular}{|c|c|c|c|c|c|}
\hline & B3LYP & BP86 & & B3LYP & BP86 \\
\hline \multirow[t]{19}{*}{$a_{1}$} & $53(0)$ & $51(1)$ & \multirow[t]{15}{*}{$\mathrm{b}_{1}$} & $18(0)$ & $18(0)$ \\
\hline & $190(0)$ & $190(0)$ & & $60(0)$ & $58(0)$ \\
\hline & $254(3)$ & 261(4) & & $290(4)$ & $301(4)$ \\
\hline & $376(2)$ & $379(1)$ & & $468(4)$ & $434(2)$ \\
\hline & $515(2)$ & $514(1)$ & & $566(0)$ & $542(0)$ \\
\hline & $587(0)$ & $557(0)$ & & $814(0)$ & $778(0)$ \\
\hline & 771(9) & $734(10)$ & & $833(0)$ & $798(0)$ \\
\hline & $789(25)$ & $758(17)$ & & $886(1)$ & $838(0)$ \\
\hline & $844(2)$ & $812(0)$ & & 994(29) & $961(27)$ \\
\hline & $894(1)$ & $848(2)$ & & 1059(0) & $1027(0)$ \\
\hline & $1031(24)$ & $1000(25)$ & & $1271(0)$ & $1220(0)$ \\
\hline & 1064(1) & 1033(1) & & $1362(0)$ & $1317(0)$ \\
\hline & $1133(0)$ & 1101(1) & & 1437(11) & 1385(11) \\
\hline & 1396(3) & $1357(2)$ & & $3235(1)$ & $3160(1)$ \\
\hline & $1480(0)$ & $1430(0)$ & & $3247(3)$ & $3172(4)$ \\
\hline & 1931(824) & $1847(643)$ & \multirow[t]{18}{*}{$\mathrm{b}_{2}$} & $95(0)$ & $99(0)$ \\
\hline & $3235(0)$ & $3160(0)$ & & $202(2)$ & 212(1) \\
\hline & $3255(1)$ & $3181(2)$ & & $258(1)$ & $268(1)$ \\
\hline & $3264(1)$ & 3189(1) & & $339(20)$ & $343(7)$ \\
\hline \multirow[t]{14}{*}{$a_{2}$} & $4(0)$ & $10 \mathrm{i}(0)$ & & $452(24)$ & $499(2)$ \\
\hline & $80(0)$ & $76(0)$ & & 592(13) & $570(9)$ \\
\hline & $316(0)$ & $324(0)$ & & $765(10)$ & $731(8)$ \\
\hline & $573(0)$ & $548(0)$ & & $785(76)$ & $754(68)$ \\
\hline & $821(0)$ & $786(0)$ & & 0839(92) & $808(79)$ \\
\hline & $832(0)$ & $799(0)$ & & 0893(1) & $847(2)$ \\
\hline & $883(0)$ & $836(0)$ & & $1030(24)$ & 999(19) \\
\hline & $994(0)$ & $961(0)$ & & 1059(44) & 1027(36) \\
\hline & 1061(0) & $1030(0)$ & & 1132(6) & 1100(6) \\
\hline & $1271(0)$ & $1220(0)$ & & 1386(176) & $1346(150)$ \\
\hline & $1369(0)$ & $1327(0)$ & & 1478(11) & $1428(12)$ \\
\hline & $1433(0)$ & $1380(0)$ & & $3235(1)$ & $3160(1)$ \\
\hline & $3235(0)$ & $3160(0)$ & & $3255(0)$ & $3180(0)$ \\
\hline & $3247(0)$ & $3171(0)$ & & $3264(2)$ & $3189(4)$ \\
\hline
\end{tabular}


Table S14. Harmonic vibrational frequencies $\left(\right.$ in $\mathrm{cm}^{-1}$ ) and infrared intensities (in parentheses, in $\mathrm{km} / \mathrm{mol}$ ) for the structure VIIIb of singlet $\mathrm{Cp}_{2} \mathrm{Ni}_{2}(\mathrm{CO})$ with $\mathrm{C}_{\mathrm{s}}$ symmetry

\begin{tabular}{|c|c|c|c|c|c|}
\hline & B3LYP & BP86 & & B3LYP & BP86 \\
\hline \multirow[t]{37}{*}{$\mathrm{a}^{\prime}$} & $47(0)$ & $51(0)$ & a" & $28 \mathrm{i}(1)$ & $34 \mathrm{i}(1)$ \\
\hline & $80(0)$ & $73(1)$ & & $24(0)$ & $19(0)$ \\
\hline & $118(1)$ & $143(0)$ & & $89(0)$ & $86(0)$ \\
\hline & $169(2)$ & $185(1)$ & & $149(1)$ & $150(1)$ \\
\hline & $187(1)$ & $243(2)$ & & $274(0)$ & $277(0)$ \\
\hline & $286(1)$ & $294(2)$ & & $285(0)$ & $295(1)$ \\
\hline & $346(35)$ & $354(6)$ & & $308(5)$ & $312(5)$ \\
\hline & $383(2)$ & $390(1)$ & & $558(1)$ & $538(1)$ \\
\hline & $404(2)$ & $413(0)$ & & $578(2)$ & $555(1)$ \\
\hline & $505(46)$ & $522(12)$ & & $775(11)$ & $733(12)$ \\
\hline & $597(4)$ & $566(0)$ & & $814(6)$ & $778(5)$ \\
\hline & $634(14)$ & $621(8)$ & & $828(0)$ & 797(0) \\
\hline & $706(4)$ & $688(4)$ & & $847(0)$ & $809(0)$ \\
\hline & 754(14) & 707(9) & & $867(1)$ & $836(1)$ \\
\hline & 781(80) & $742(88)$ & & $894(1)$ & $852(0)$ \\
\hline & 799(18) & $764(13)$ & & 944(9) & $912(8)$ \\
\hline & $815(36)$ & $770(5)$ & & 993(11) & $966(10)$ \\
\hline & $839(10)$ & $803(2)$ & & $1055(0)$ & $1014(0)$ \\
\hline & $895(3)$ & $852(6)$ & & $1060(0)$ & $1027(0)$ \\
\hline & $907(2)$ & $863(1)$ & & $1257(0)$ & $1204(0)$ \\
\hline & $998(2)$ & $972(6)$ & & 1271(0) & $1219(0)$ \\
\hline & $1028(12)$ & 994(11) & & $1338(3)$ & $1284(1)$ \\
\hline & $1037(3)$ & 1004(2) & & $1369(0)$ & $1322(0)$ \\
\hline & 1064(8) & 1032(4) & & 1409(4) & $1347(6)$ \\
\hline & 1101(11) & 1065(8) & & $1429(4)$ & $1376(5)$ \\
\hline & 1132(1) & 1097(3) & & $3203(1)$ & $3128(0)$ \\
\hline & $1368(36)$ & $1322(26)$ & & $3227(3)$ & $3145(6)$ \\
\hline & $1388(13)$ & $1346(12)$ & & $3231(0)$ & $3157(0)$ \\
\hline & 1466(10) & 1411(6) & & $3244(2)$ & $3170(2)$ \\
\hline & 1490(6) & 1427(4) & & & \\
\hline & 2034(1608) & 1947(1301) & & & \\
\hline & $3212(0)$ & 3130(0) & & & \\
\hline & $3220(0)$ & 3142(1) & & & \\
\hline & $3236(1)$ & $3155(4)$ & & & \\
\hline & $3237(2)$ & $3161(1)$ & & & \\
\hline & $3254(1)$ & $3180(1)$ & & & \\
\hline & $3269(2)$ & 3194(2) & & & \\
\hline
\end{tabular}


Table S15. Harmonic vibrational frequencies (in $\mathrm{cm}^{-1}$ ) and infrared intensities (in parentheses, in $\mathrm{km} / \mathrm{mol}$ ) for the structure VIIIc of triplet $\mathrm{Cp}_{2} \mathrm{Ni}_{2}(\mathrm{CO})$ with $\mathrm{C}_{\mathrm{s}}$ symmetry

\begin{tabular}{|c|c|c|c|c|c|}
\hline & B3LYP & BP86 & & B3LYP & BP86 \\
\hline \multirow[t]{37}{*}{$a^{\prime}$} & $48(0)$ & $26(8)$ & a" & $4 \mathrm{i}(0)$ & $20(0)$ \\
\hline & $84(1)$ & $57(0)$ & & $21(0)$ & $25(0)$ \\
\hline & $127(0)$ & $178(0)$ & & $60(0)$ & $59(0)$ \\
\hline & 177(1) & $182(4)$ & & $105(0)$ & $112(0)$ \\
\hline & $255(0)$ & $238(0)$ & & $247(1)$ & $258(2)$ \\
\hline & $287(5)$ & $276(4)$ & & $278(2)$ & $272(0)$ \\
\hline & $317(6)$ & $333(8)$ & & $454(4)$ & $428(2)$ \\
\hline & $361(30)$ & $354(4)$ & & $594(0)$ & $565(0)$ \\
\hline & $430(16)$ & $355(0)$ & & $602(0)$ & $569(1)$ \\
\hline & $562(23)$ & $495(4)$ & & 791(0) & $755(0)$ \\
\hline & $595(0)$ & $564(0)$ & & $795(0)$ & $758(0)$ \\
\hline & $598(2)$ & $555(2)$ & & $842(0)$ & $798(0)$ \\
\hline & 790(129) & $752(65)$ & & $845(0)$ & $813(0)$ \\
\hline & 796(44) & $760(12)$ & & $881(0)$ & $834(0)$ \\
\hline & $800(2)$ & $760(60)$ & & $887(0)$ & $839(1)$ \\
\hline & $808(18)$ & 764(9) & & $1017(15)$ & $988(0)$ \\
\hline & $843(8)$ & $803(1)$ & & $1022(22)$ & $989(35)$ \\
\hline & $846(0)$ & $813(0)$ & & $1065(0)$ & $1032(0)$ \\
\hline & $885(0)$ & $844(0)$ & & $1066(0)$ & $1037(0)$ \\
\hline & $891(0)$ & $845(1)$ & & $1269(0)$ & $1221(0)$ \\
\hline & 1013(10) & $982(3)$ & & 1273(0) & $1221(0)$ \\
\hline & 1018(9) & 983(24) & & 1374(0) & $1337(0)$ \\
\hline & 1062(2) & $1025(2)$ & & 1381(0) & $1344(0)$ \\
\hline & 1065(4) & $1035(1)$ & & $1453(1)$ & $1405(0)$ \\
\hline & 1131(1) & 1102(4) & & 1459(2) & $1406(3)$ \\
\hline & 1136(1) & $1103(0)$ & & 3233(0) & 3161(0) \\
\hline & $1375(0)$ & $1323(10)$ & & $3235(0)$ & $3162(0)$ \\
\hline & $1380(7)$ & $1340(0)$ & & $3249(0)$ & $3175(0)$ \\
\hline & 1449(0) & $1400(1)$ & & $3250(2)$ & $3175(3)$ \\
\hline & 1994(873) & $1861(562)$ & & & \\
\hline & $1455(2)$ & 1402(4) & & & \\
\hline & $3235(0)$ & 3161(0) & & & \\
\hline & $3236(0)$ & $3161(0)$ & & & \\
\hline & $3248(0)$ & $3175(1)$ & & & \\
\hline & $3249(2)$ & 3175(3) & & & \\
\hline & 3261(1) & 3187(4) & & & \\
\hline & $3262(3)$ & $3187(1)$ & & & \\
\hline
\end{tabular}


Table S16. Cartesian coordinates for the 15 structures discussed in this paper $\mathrm{Cp}_{2} \mathrm{Fe}_{2}(\mu-\mathrm{NO})_{2}(\mathbf{I a})$

$\begin{array}{lrrr}6 & -1.163509 & 2.887184 & 0.379055 \\ 6 & 0.000000 & 2.871325 & 1.230358 \\ 6 & 1.163509 & 2.887184 & 0.379055 \\ 6 & 0.722201 & 2.871610 & -0.990224 \\ 6 & -0.722201 & 2.871610 & -0.990224 \\ 1 & -2.201889 & 2.811203 & 0.712532 \\ 1 & 0.000000 & 2.847537 & 2.323377 \\ 1 & 2.201889 & 2.811203 & 0.712532 \\ 1 & 1.366256 & 2.825693 & -1.872634 \\ 1 & -1.366256 & 2.825693 & -1.872634 \\ 26 & 0.000000 & 1.169347 & 0.005019 \\ 26 & 0.000000 & -1.169347 & 0.005019 \\ 7 & 1.357482 & 0.000000 & -0.002475 \\ 7 & -1.357482 & 0.000000 & -0.002475 \\ 8 & 2.588005 & 0.000000 & -0.020561 \\ 8 & -2.588005 & 0.000000 & -0.020561 \\ 6 & 1.163509 & -2.887184 & 0.379055 \\ 6 & 0.000000 & -2.871325 & 1.230358 \\ 6 & -1.163509 & -2.887184 & 0.379055 \\ 6 & -0.722201 & -2.871610 & -0.990224 \\ 6 & 0.722201 & -2.871610 & -0.990224 \\ 1 & 2.201889 & -2.811203 & 0.712532 \\ 1 & 0.000000 & -2.847537 & 2.323377 \\ 1 & -2.201889 & -2.811203 & 0.712532 \\ 1 & -1.366256 & -2.825693 & -1.872634 \\ 1 & 1.366256 & -2.825693 & -1.872634\end{array}$

\section{$\mathrm{Cp}_{2} \mathrm{Fe}_{2}(\mathrm{NO})_{2}(\mathbf{I b})$}

$\begin{array}{rrrr}26 & 0.000000 & 1.163054 & 0.000000 \\ 26 & 0.000000 & -1.163054 & 0.000000 \\ 6 & 0.620036 & 3.096968 & 0.720463 \\ 6 & -0.620036 & -3.096968 & -0.720463 \\ 6 & -0.620036 & -3.096968 & 0.720463 \\ 6 & 0.620036 & 3.096968 & -0.720463 \\ 6 & -2.140943 & -1.496881 & 0.000000 \\ 6 & 2.140943 & 1.496881 & 0.000000 \\ 6 & -1.549216 & -2.098619 & 1.167773 \\ 6 & 1.549216 & 2.098619 & -1.167773 \\ 6 & 1.549216 & 2.098619 & 1.167773 \\ 6 & -1.549216 & -2.098619 & -1.167773 \\ 1 & -0.024366 & 3.706721 & 1.359738 \\ 1 & 0.024366 & -3.706721 & 1.359738 \\ 1 & -0.024366 & 3.706721 & -1.359738 \\ 1 & 0.024366 & -3.706721 & -1.359738 \\ 1 & -2.926628 & -0.741313 & 0.000000 \\ 1 & 2.926628 & 0.741313 & 0.000000 \\ 1 & 1.769627 & 1.842585 & 2.207270 \\ 1 & -1.769627 & -1.842585 & -2.207270 \\ 1 & -1.769627 & -1.842585 & 2.207270 \\ 1 & 1.769627 & 1.842585 & -2.207270 \\ 7 & -1.615315 & 1.313781 & 0.000000 \\ 7 & 1.615315 & -1.313781 & 0.000000 \\ 8 & -2.761043 & 1.681103 & 0.000000\end{array}$




\section{$\mathrm{Cp}_{2} \mathrm{Fe}_{2}(\mu-\mathrm{NO})$ (IIa)}

$\begin{array}{cccc}6 & 0.000000 & 3.126316 & 0.735550 \\ 6 & -1.160478 & 2.849009 & -0.058096 \\ 6 & -0.725745 & 2.425781 & -1.369470 \\ 6 & 0.725745 & 2.425781 & -1.369470 \\ 6 & 1.160478 & 2.849009 & -0.058096 \\ 1 & 0.000000 & 3.401125 & 1.793740 \\ 1 & -2.198694 & 2.913857 & 0.279554 \\ 1 & -1.373741 & 2.167227 & -2.210755 \\ 1 & 1.373741 & 2.167227 & -2.210755 \\ 1 & 2.198694 & 2.913857 & 0.279554 \\ 26 & 0.000000 & 0.993964 & -0.043926 \\ 26 & 0.000000 & -0.993964 & -0.043926 \\ 6 & 0.000000 & -3.126316 & 0.735550 \\ 6 & -1.160478 & -2.849009 & -0.058096 \\ 6 & -0.725745 & -2.425781 & -1.369470 \\ 6 & 0.725745 & -2.425781 & -1.369470 \\ 6 & 1.160478 & -2.849009 & -0.058096 \\ 1 & 0.000000 & -3.401125 & 1.793740 \\ 1 & -2.198694 & -2.913857 & 0.279554 \\ 1 & -1.373741 & -2.167227 & -2.210755 \\ 1 & 1.373741 & -2.167227 & -2.210755 \\ 1 & 2.198694 & -2.913857 & 0.279554 \\ 7 & 0.000000 & 0.000000 & 1.473576 \\ 8 & 0.000000 & 0.000000 & 2.692678\end{array}$

\section{$\mathrm{Cp}_{2} \mathrm{Fe}_{2}(\mathrm{NO})(\mathbf{I I b})$}

$\begin{array}{cccc}6 & 0.784893 & -2.920927 & 0.000000 \\ 6 & 1.046152 & -2.121454 & 1.162114 \\ 6 & 1.641898 & -0.852163 & 0.728890 \\ 6 & 1.641898 & -0.852163 & -0.728890 \\ 6 & 1.046152 & -2.121454 & -1.162114 \\ 1 & 0.322682 & -3.911990 & 0.000000 \\ 1 & 0.925354 & -2.427910 & 2.204370 \\ 1 & 2.215913 & -0.191411 & 1.386185 \\ 1 & 2.215913 & -0.191411 & -1.386185 \\ 1 & 0.925354 & -2.427910 & -2.204370 \\ 26 & -0.448854 & -1.153852 & 0.000000 \\ 26 & 0.254195 & 0.830574 & 0.000000 \\ 6 & -1.158111 & 2.385316 & 0.000000 \\ 6 & -0.315993 & 2.500379 & 1.163415 \\ 6 & 1.046152 & 2.664645 & 0.723558 \\ 6 & 1.046152 & 2.664645 & -0.723558 \\ 6 & -0.315993 & 2.500379 & -1.163415 \\ 1 & -2.236301 & 2.206313 & 0.000000 \\ 1 & -0.652792 & 2.410590 & 2.200276 \\ 1 & 1.923821 & 2.775917 & 1.366970 \\ 1 & 1.923821 & 2.775917 & -1.366970 \\ 1 & -0.652792 & 2.410590 & -2.200276 \\ 7 & -2.065988 & -1.158851 & 0.000000 \\ 8 & -3.270892 & -1.249341 & 0.000000\end{array}$


$\mathrm{Cp}_{2} \mathrm{Co}_{2}(\mu-\mathrm{NO})_{2}($ III $)$

$\begin{array}{rrrr}6 & -1.160281 & 2.777845 & 0.784663 \\ 6 & 0.000000 & 2.587156 & 1.607868 \\ 6 & 1.160281 & 2.777845 & 0.784663 \\ 6 & 0.717931 & 3.118039 & -0.552895 \\ 6 & -0.717931 & 3.118039 & -0.552895 \\ 1 & -2.200663 & 2.656343 & 1.098168 \\ 1 & 0.000000 & 2.297286 & 2.661883 \\ 1 & 2.200663 & 2.656343 & 1.098168 \\ 1 & 1.364683 & 3.307812 & -1.413636 \\ 1 & -1.364683 & 3.307812 & -1.413636 \\ 27 & 0.000000 & 1.212752 & -0.082462 \\ 27 & 0.000000 & -1.212752 & -0.082462 \\ 7 & 1.213586 & 0.000000 & -0.619050 \\ 7 & -1.213586 & 0.000000 & -0.619050 \\ 8 & 2.380218 & 0.000000 & -0.987443 \\ 8 & -2.380218 & 0.000000 & -0.987443 \\ 6 & 1.160281 & -2.777845 & 0.784663 \\ 6 & 0.000000 & -2.587156 & 1.607868 \\ 6 & -1.160281 & -2.777845 & 0.784663 \\ 6 & -0.717931 & -3.118039 & -0.552895 \\ 6 & 0.717931 & -3.118039 & -0.552895 \\ 1 & 2.200663 & -2.656343 & 1.098168 \\ 1 & 0.000000 & -2.297286 & 2.661883 \\ 1 & -2.200663 & -2.656343 & 1.098168 \\ 1 & -1.364683 & -3.307812 & -1.413636 \\ 1 & 1.364683 & -3.307812 & -1.413636\end{array}$

\section{$\mathrm{Cp}_{2} \mathrm{Co}_{2}(\mu-\mathrm{NO})(\mathbf{I V a})$}

$\begin{array}{rrrr}6 & 0.000000 & 3.202753 & 0.597485 \\ 6 & -1.157750 & 2.839505 & -0.166812 \\ 6 & -0.718370 & 2.309788 & -1.444864 \\ 6 & 0.718370 & 2.309788 & -1.444864 \\ 6 & 1.157750 & 2.839505 & -0.166812 \\ 1 & 0.000000 & 3.585604 & 1.621264 \\ 1 & -2.197580 & 2.934726 & 0.159179 \\ 1 & -1.369238 & 1.962944 & -2.251414 \\ 1 & 1.369238 & 1.962944 & -2.251414 \\ 1 & 2.197580 & 2.934726 & 0.159179 \\ 27 & 0.000000 & 1.074311 & 0.079887 \\ 27 & 0.000000 & -1.074311 & 0.079887 \\ 6 & 0.000000 & -3.202753 & 0.597485 \\ 6 & -1.157750 & -2.839505 & -0.166812 \\ 6 & -0.718370 & -2.309788 & -1.444864 \\ 6 & 0.718370 & -2.309788 & -1.444864 \\ 6 & 1.157750 & -2.839505 & -0.166812 \\ 1 & 0.000000 & -3.585604 & 1.621264 \\ 1 & -2.197580 & -2.934726 & 0.159179 \\ 1 & -1.369238 & -1.962944 & -2.251414 \\ 1 & 1.369238 & -1.962944 & -2.251414 \\ 1 & 2.197580 & -2.934726 & 0.159179 \\ 7 & 0.000000 & 0.000000 & 1.502549 \\ 8 & 0.000000 & 0.000000 & 2.725632\end{array}$




\section{$\mathrm{Cp}_{2} \mathrm{Co}_{2}(\mathrm{NO})(\mathbf{I V b})$}

$\begin{array}{rrcc}27 & -0.144393 & -1.340373 & 0.000000 \\ 27 & 0.000000 & 1.056505 & 0.000000 \\ 6 & -1.932041 & -2.304408 & 0.723098 \\ 6 & 0.369777 & 2.997005 & -0.731172 \\ 6 & 0.369777 & 2.997005 & 0.731172 \\ 6 & -1.932041 & -2.304408 & -0.723098 \\ 6 & 1.971948 & 1.485822 & 0.000000 \\ 6 & -1.734077 & -0.108081 & 0.000000 \\ 6 & 1.380026 & 2.094301 & 1.181096 \\ 6 & -1.851835 & -0.949843 & -1.185122 \\ 6 & -1.851835 & -0.949843 & 1.185122 \\ 6 & 1.380026 & 2.094301 & -1.181096 \\ 1 & -1.958913 & -3.194213 & 1.357652 \\ 1 & -0.306177 & 3.577977 & 1.365585 \\ 1 & -1.958913 & -3.194213 & -1.357652 \\ 1 & -0.306177 & 3.577977 & -1.365585 \\ 1 & 2.757683 & 0.725647 & 0.000000 \\ 1 & -1.786623 & 1.041321 & 0.000000 \\ 1 & -1.832175 & -0.604996 & 2.221137 \\ 1 & 1.622456 & 1.852320 & -2.218992 \\ 1 & 1.622456 & 1.852320 & 2.218992 \\ 1 & -1.832175 & -0.604996 & -2.221137 \\ 7 & 1.427305 & -1.726821 & 0.000000 \\ 8 & 2.608462 & -1.948505 & 0.000000\end{array}$

\section{$\mathrm{Cp}_{2} \mathrm{Ni}_{2}(\mu-\mathrm{NO})_{2}(\mathbf{V})$}

$\begin{array}{rrrr}6 & 2.360920 & 0.386120 & 1.614237 \\ 6 & 2.408490 & -1.009549 & 1.235135 \\ 6 & 2.999143 & -1.097043 & -0.069182 \\ 6 & 3.283970 & 0.244615 & -0.517724 \\ 6 & 2.911454 & 1.156175 & 0.539707 \\ 1 & 1.959706 & 0.782885 & 2.550770 \\ 1 & 2.051062 & -1.851169 & 1.834475 \\ 1 & 3.148591 & -2.012379 & -0.647590 \\ 1 & 3.723234 & 0.522133 & -1.479804 \\ 1 & 2.975761 & 2.246544 & 0.495301 \\ 28 & 1.185361 & 0.055419 & -0.199989 \\ 28 & -1.185360 & 0.055419 & -0.199989 \\ 7 & 0.000000 & -1.221879 & -1.113334 \\ 7 & 0.000000 & 1.301330 & -0.853368 \\ 8 & 0.000000 & -2.420073 & -0.877704 \\ 8 & 0.000000 & 2.520140 & -0.893056 \\ 6 & -2.999142 & -1.097044 & -0.069181 \\ 6 & -2.408490 & -1.009547 & 1.235137 \\ 6 & -2.360921 & 0.386122 & 1.614236 \\ 6 & -2.911455 & 1.156175 & 0.539704 \\ 6 & -3.283970 & 0.244613 & -0.517725 \\ 1 & -3.148589 & -2.012382 & -0.647587 \\ 1 & -2.051062 & -1.851166 & 1.834479 \\ 1 & -1.959709 & 0.782889 & 2.550769 \\ 1 & -2.975763 & 2.246543 & 0.495296 \\ 1 & -3.723233 & 0.522128 & -1.479807\end{array}$




\section{$\mathrm{Cp}_{2} \mathrm{Ni}_{2}(\mu-\mathrm{NO})(\mathrm{VI})$}

$\begin{array}{rrrr}6 & 0.000000 & 3.275785 & 0.494415 \\ 6 & -1.163236 & 2.889989 & -0.260438 \\ 6 & -0.717196 & 2.280460 & -1.495000 \\ 6 & 0.717196 & 2.280460 & -1.495000 \\ 6 & 1.163236 & 2.889989 & -0.260438 \\ 1 & 0.000000 & 3.746843 & 1.480822 \\ 1 & -2.203603 & 3.023957 & 0.048661 \\ 1 & -1.361454 & 1.874343 & -2.279121 \\ 1 & 1.361454 & 1.874343 & -2.279121 \\ 1 & 2.203603 & 3.023957 & 0.048661 \\ 28 & 0.000000 & 1.149765 & 0.173353 \\ 28 & 0.000000 & -1.149765 & 0.173353 \\ 6 & 0.000000 & -3.275785 & 0.494415 \\ 6 & -1.163236 & -2.889989 & -0.260438 \\ 6 & -0.717196 & -2.280460 & -1.495000 \\ 6 & 0.717196 & -2.280460 & -1.495000 \\ 6 & 1.163236 & -2.889989 & -0.260438 \\ 1 & 0.000000 & -3.746843 & 1.480822 \\ 1 & -2.203603 & -3.023957 & 0.048661 \\ 1 & -1.361454 & -1.874343 & -2.279121 \\ 1 & 1.361454 & -1.874343 & -2.279121 \\ 1 & 2.203603 & -3.023957 & 0.048661 \\ 7 & 0.000000 & 0.000000 & 1.511472 \\ 8 & 0.000000 & 0.000000 & 2.733702\end{array}$

\section{$\mathrm{Cp}_{2} \mathrm{Ni}_{2}(\mu-\mathrm{CO})_{2}(\mathbf{V I I a})$}

$\begin{array}{rrrr}6 & -0.675215 & 3.052857 & 0.729968 \\ 6 & 0.675215 & 2.947524 & 1.177762 \\ 6 & 1.506839 & 2.824098 & 0.000000 \\ 6 & 0.675215 & 2.947524 & -1.177762 \\ 6 & -0.675215 & 3.052857 & -0.729968 \\ 1 & -1.565325 & 3.117398 & 1.361435 \\ 1 & 1.018862 & 2.912605 & 2.214435 \\ 1 & 2.591220 & 2.681893 & 0.000000 \\ 1 & 1.018862 & 2.912605 & -2.214435 \\ 1 & -1.565325 & 3.117398 & -1.361435 \\ 28 & 0.134473 & 1.202678 & 0.000000 \\ 28 & -0.134473 & -1.202678 & 0.000000 \\ 6 & 1.414844 & -0.152314 & 0.000000 \\ 6 & -1.414844 & 0.152314 & 0.000000 \\ 8 & 2.601724 & -0.291888 & 0.000000 \\ 8 & -2.601724 & 0.291888 & 0.000000 \\ 6 & 0.675215 & -3.052857 & 0.729968 \\ 6 & -0.675215 & -2.947524 & 1.177762 \\ 6 & -1.506839 & -2.824098 & 0.000000 \\ 6 & -0.675215 & -2.947524 & -1.177762 \\ 6 & 0.675215 & -3.052857 & -0.729968 \\ 1 & 1.565325 & -3.117398 & 1.361435 \\ 1 & -1.018862 & -2.912605 & 2.214435 \\ 1 & -2.591220 & -2.681893 & 0.000000 \\ 1 & -1.018862 & -2.912605 & -2.214435 \\ 1 & 1.565325 & -3.117398 & -1.361435\end{array}$




\section{$\mathrm{Cp}_{2} \mathrm{Ni}_{2}(\mu-\mathrm{CO})_{2}(\mathbf{V I I b})$}

$\begin{array}{rrrr}6 & -0.682803 & 3.089801 & 0.717815 \\ 6 & 0.682803 & 2.951090 & 1.162900 \\ 6 & 1.526502 & 2.895172 & 0.000000 \\ 6 & 0.682803 & 2.951090 & -1.162900 \\ 6 & -0.682803 & 3.089801 & -0.717815 \\ 1 & -1.565491 & 3.151668 & 1.359625 \\ 1 & 1.018606 & 2.908408 & 2.202728 \\ 1 & 2.611570 & 2.764399 & 0.000000 \\ 1 & 1.018606 & 2.908408 & -2.202728 \\ 1 & -1.565491 & 3.151668 & -1.359625 \\ 28 & 0.127679 & 1.183667 & 0.000000 \\ 28 & -0.127679 & -1.183667 & 0.000000 \\ 6 & 1.512567 & -0.157177 & 0.000000 \\ 6 & -1.512567 & 0.157177 & 0.000000 \\ 8 & 2.694855 & -0.297912 & 0.000000 \\ 8 & -2.694855 & 0.297912 & 0.000000 \\ 6 & 0.682803 & -3.089801 & 0.717815 \\ 6 & -0.682803 & -2.951090 & 1.162900 \\ 6 & -1.526502 & -2.895172 & 0.000000 \\ 6 & -0.682803 & -2.951090 & -1.162900 \\ 6 & 0.682803 & -3.089801 & -0.717815 \\ 1 & 1.565491 & -3.151668 & 1.359625 \\ 1 & -1.018606 & -2.908408 & 2.202728 \\ 1 & -2.611570 & -2.764399 & 0.000000 \\ 1 & -1.018606 & -2.908408 & -2.202728 \\ 1 & 1.565491 & -3.151668 & -1.359625\end{array}$

\section{$\mathrm{Cp}_{2} \mathrm{Ni}_{2}(\mathrm{CO})_{2}($ VIIc)}

$\begin{array}{rrrc}28 & 1.367497 & 0.278630 & 0.068002 \\ 28 & -1.027128 & -0.420555 & 0.122845 \\ 6 & 1.985857 & -1.086232 & -1.388012 \\ 6 & -1.949225 & 1.745581 & 0.077817 \\ 6 & -0.917943 & 1.468974 & 1.041350 \\ 6 & 1.029994 & -0.198404 & -2.009816 \\ 6 & -0.021699 & 2.725492 & -0.722650 \\ 6 & -0.157517 & -2.007463 & -1.103546 \\ 6 & 0.331912 & 2.059960 & 0.519517 \\ 6 & -0.294085 & -0.795501 & -1.879066 \\ 6 & 1.246974 & -2.163935 & -0.790550 \\ 6 & -1.380071 & 2.504620 & -1.000480 \\ 1 & 3.076948 & -1.023135 & -1.460513 \\ 1 & -1.086011 & 1.236208 & 2.100783 \\ 1 & 1.263339 & 0.650436 & -2.659476 \\ 1 & -3.014551 & 1.526408 & 0.206904 \\ 1 & 0.678714 & 3.288534 & -1.349468 \\ 1 & -0.894762 & -2.812498 & -1.034539 \\ 1 & 1.679025 & -2.984797 & -0.209832 \\ 1 & -1.917875 & 2.831301 & -1.896800 \\ 1 & 1.098427 & 2.467630 & 1.195140 \\ 1 & -1.173180 & -0.489005 & -2.456575 \\ 6 & 2.128965 & -0.074528 & 1.645107 \\ 6 & -2.449030 & -1.290484 & 0.764962 \\ 8 & 2.544316 & -0.386305 & 2.696421 \\ 8 & -3.364964 & -1.869400 & 1.215190\end{array}$




\section{$\mathrm{Cp}_{2} \mathrm{Ni}_{2}(\mu-\mathrm{CO})$ (VIIIa)}

$\begin{array}{rrrc}6 & 0.000000 & 3.233919 & 0.540792 \\ 6 & -1.154007 & 2.845226 & -0.217670 \\ 6 & -0.709299 & 2.308193 & -1.502286 \\ 6 & 0.709299 & 2.308193 & -1.502286 \\ 6 & 1.154007 & 2.845226 & -0.217670 \\ 1 & 0.000000 & 3.660032 & 1.546772 \\ 1 & -2.195626 & 2.951530 & 0.099501 \\ 1 & -1.360931 & 1.942432 & -2.299858 \\ 1 & 1.360931 & 1.942432 & -2.299858 \\ 1 & 2.195626 & 2.951530 & 0.099501 \\ 28 & 0.000000 & 1.115465 & 0.146300 \\ 28 & 0.000000 & -1.115465 & 0.146300 \\ 6 & 0.000000 & -3.233919 & 0.540792 \\ 6 & -1.154007 & -2.845226 & -0.217670 \\ 6 & -0.709299 & -2.308193 & -1.502286 \\ 6 & 0.709299 & -2.308193 & -1.502286 \\ 6 & 1.154007 & -2.845226 & -0.217670 \\ 1 & 0.000000 & -3.660032 & 1.546772 \\ 1 & -2.195626 & -2.951530 & 0.099501 \\ 1 & -1.360931 & -1.942432 & -2.299858 \\ 1 & 1.360931 & -1.942432 & -2.299858 \\ 1 & 2.195626 & -2.951530 & 0.099501 \\ 6 & 0.000000 & 0.000000 & 1.622288 \\ 8 & 0.000000 & 0.000000 & 2.821349\end{array}$

\section{$\mathrm{Cp}_{2} \mathrm{Ni}_{2}(\mu-\mathrm{CO})($ VIIIb)}

$\begin{array}{rrrr}6 & 0.618130 & 3.242441 & 0.000000 \\ 6 & -0.163909 & 2.930398 & 1.165468 \\ 6 & -1.430539 & 2.407416 & 0.725046 \\ 6 & -1.430539 & 2.407416 & -0.725046 \\ 6 & -0.163909 & 2.930398 & -1.165468 \\ 1 & 1.643310 & 3.622685 & 0.000000 \\ 1 & 0.158951 & 3.040898 & 2.204243 \\ 1 & -2.251873 & 2.080576 & 1.368139 \\ 1 & -2.251873 & 2.080576 & -1.368139 \\ 1 & 0.158951 & 3.040898 & -2.204243 \\ 28 & 0.073727 & 1.103164 & 0.000000 \\ 28 & 0.075663 & -1.103821 & 0.000000 \\ 6 & 0.617803 & -3.243394 & 0.000000 \\ 6 & -0.163909 & -2.930488 & 1.165443 \\ 6 & -1.430117 & -2.406272 & 0.725013 \\ 6 & -1.430117 & -2.406272 & -0.725013 \\ 6 & -0.163909 & -2.930488 & -1.165443 \\ 1 & 1.642519 & -3.624883 & 0.000000 \\ 1 & 0.158800 & -3.041360 & 2.204226 \\ 1 & -2.251145 & -2.078728 & 1.368134 \\ 1 & -2.251145 & -2.078728 & -1.368134 \\ 1 & 0.158800 & -3.041360 & -2.204226 \\ 6 & 1.586499 & -0.000489 & 0.000000 \\ 8 & 2.778608 & 0.001730 & 0.000000\end{array}$




\section{$\mathrm{Cp}_{2} \mathrm{Ni}_{2}(\mathrm{CO})$ (VIIIc)}

$\begin{array}{rrcc}28 & -0.127732 & -1.435076 & 0.000000 \\ 28 & 0.000000 & 1.058004 & 0.000000 \\ 6 & -1.957127 & -0.782575 & 0.710928 \\ 6 & 1.475084 & 2.515579 & -0.708750 \\ 6 & 1.475084 & 2.515579 & 0.708750 \\ 6 & -1.957127 & -0.782575 & -0.710928 \\ 6 & 2.008805 & 0.327881 & 0.000000 \\ 6 & -1.902256 & 1.501787 & 0.000000 \\ 6 & 1.751229 & 1.156727 & 1.154107 \\ 6 & -1.747742 & 0.629270 & -1.142968 \\ 6 & -1.747742 & 0.629270 & 1.142968 \\ 6 & 1.751229 & 1.156727 & -1.154107 \\ 1 & -2.335698 & -1.570540 & 1.371389 \\ 1 & 1.263533 & 3.367280 & 1.361027 \\ 1 & -2.335698 & -1.570540 & -1.371389 \\ 1 & 1.263533 & 3.367280 & -1.361027 \\ 1 & 2.387266 & -0.695822 & 0.000000 \\ 1 & -2.039474 & 2.588606 & 0.000000 \\ 1 & -1.773104 & 0.956173 & 2.188280 \\ 1 & 1.820096 & 0.831573 & -2.196286 \\ 1 & 1.820096 & 0.831573 & 2.196286 \\ 1 & -1.773104 & 0.956173 & -2.188280 \\ 6 & 0.572200 & -3.039389 & 0.000000 \\ 8 & 0.868654 & -4.184180 & 0.000000\end{array}$

